\title{
UM MÉTODO DE ANÁLISE DO RACIOCINIO APLICADO EM ENFERMAGEM EM NEUROCIÊNCIA
}

\author{
Maria Sumie Koizumi* \\ Marly Theoto Rocha** \\ Catarina Osawa*** \\ Mirna Namie Okamura****
}

KOIZUMI, M.S. et al. Um método de análise do raciocínio diagnóstico aplicado em enfermagem em neurociência. Rev, Esc. Enf. USP., v. 27, n. 1, p. 161-73 , abr. 1993.

Resumo: Trata-se de um estudo preliminar que testa a aplicabilidade de um método de caracterização do conhecimento fundamentado na teoria dos grafos. Os grafos para elucidação do processo diagnóstico foram construídos por 11 (onze) enfermeiras de uma UTI geral as quais usualmente assistem pacientes com problemas neurológicos. Os resultados obtidos mostram que: a estrutura de conhecimento encontra-se em desenvolvimento; há consenso quanto à formulação dos diagnósticos; e, a base de dados que a fundamenta é diversificada e está ligada à forma de especificação da relação causual. O método mostrou-se viável e deve ser reaplicado em uma população maior.

UNITERMOS: Enfermagem neurológica. Diagnóstico de Enfermagem.

\section{Introduçāo}

O documento básico sobre diagnóstico de enfermagem em Enfermagem em Neurociência data de 1988. Nele estāo descritos alguns diagnósticos específicos com suas características definidoras, as respectivas intervenções de enfermagem e os resultados esperados classificados em seis grandes categorias diagnósticas.

São elas: consciência e cognição; comunicação; motricidade; sensibilidade; sono e repouso; e, sexualidade ${ }^{1 .}$

Neste mesmo documento ${ }^{1}$, seus autores ressaltam que ele retrata o estado do conhecimento da enfermagem em neurociência naquela data e que estudos que validem os diagnósticos propostos são necessários.

Pesquisas para validação de diagnósticos específicos de enfermagem em neurociência, questionando a terminologia adequada ou determinando as características definidoras e as intervenções de enfermagem tem sido realizados

\footnotetext{
* Enfermeira. Professora Associada do Departamen Enfermagem da USP.

* Enfermeira. Professora Doutora do Departamento de Enfermagem de Saúde Coletiva da Escola de Enfermagem da USP.

*** Enfermeira.do Hospital Alemäo "Oswaldo Cruz".

**** Enfermeira da Secretaria Municipal de Saúde de São Paulo.
} 
nos últimos anos. Contudo, lacunas quanto ao raciocínio diagnóstico desenvolvido pelo enfermeiro enquanto processo até a definiçăo do diagnóstico persistem.

Numa dada especialidade, espera-se que a base de dados dessa disciplina seja estruturada c documentada. Na enfermagem em neurociência, julga-se que cla encontra-se ainda em fase de desenvolvimento. De certa forma, pode-se dizer que tanto a estrutura como a documentação acerca de sua base de conhecimentos continua nebulosa.

Um método de caracterização do conhecimento utilizando a tcoria dos grafos tem sido desenvolvido com resultados promissores por GRECO; RO$\mathrm{CHA}^{4}$, ROCHA; ROCHA ${ }^{10}$ e ROCHA ${ }^{11}$. LEÇO $^{5}$ por sua vez, utilizou esta metodologia para representar e analisar o conhecimento médico que fundamenta o diagnóstico de doenças cardíacas congênitas entre médicos especialistas c não especialistas da área. Uma das premissas deste estudo foi a existência do conhecimento estruturado e documentado.

Ora, se o diagnóstico de enfermagem vem sendo feito na prática clínica, acredita-se que utilizando este método seja possível extrair o conhecimento que o enfermeiro utiliza para defini-lo e principalmente, esclarecer em que estágio de estruturação cle se encontra.

Considerando-se que, o preparo dos enfermeiros, tipo de pacientes e de unidades em que são assistidos seriam fatores importantes para a análise do raciocínio diagnóstico, optou-se por limitar o estudo a uma realidade em que eles fossem mais uniformes. Assim, como em algumas unidades de terapia intensiva (UTIs), os enfermeiros, rotineiramente, fazem diagnósticos de enfermagem e como nessas unidades, a presença de pacientes com pressão intracraniana aumentada é usual, este foi o limite estabelecido.

Com isto, pretendeu-se analisar a estrutura do conhecimento e o raciocínio diagnóstico utilizado pelos enfermeiros de UTI ao elaborar diagnósticos de enfermagem específicos de pacientes ali admitidos devido desequilíbrio no conteúdo intracraniano.

\section{MATERIAL E MÉTODO}

\subsection{População}

Foi constituida por enfermeiras de uma UTI geral de um hospital da rede privada de São Paulo. A seleção desta UTI deveu-se ao fato de suas enfermeiras utilizarem o diagnóstico de enfermagem.

O diagnóstico de enfermagem foi implantado nesta Unidade a partir de 1987 por uma de suas enfermeiras, a qual fez um curso de especialização em enfermagem em terapia intensiva, no exterior e vivenciou essa prática durante seu curso. Esta mesma enfermeira foi responsável pela orientação e reciclagem das enfermeiras desta Unidade. 
Os critérios de inclusão da enfermeira no estudo foram:

- consentir na participação voluntária,

- ter concluido a fase de treinamento para fazer diagnósticos de enfermagem e estar utilizando-os na prática diária.

\subsection{Método}

O método utilizado foi proposto por LEÃO ${ }^{5}$ e MACHADO et al ${ }^{6} \mathrm{com}$ as devidas adaptações para este estudo. Assim, a aquisição do conhecimento foi feita por meio de entrevista individual na qual solicitava-se à enfermeira enunciar por escrito, um diagnóstico de enfermagem específico para pacientes que apresentem desequilíbrio no conteúdo intracraniano. A seguir, pedia-se a lista de dados por ela julgados como necessários para a formulação daquele diagnóstico e sua ordenação por prioridade, da mais importante para a menos importante.

O passo seguinte foi a construção do grafo, cujo modelo pode ser visto na Figura 1.

Figura 1: MODELO DE GRAFO

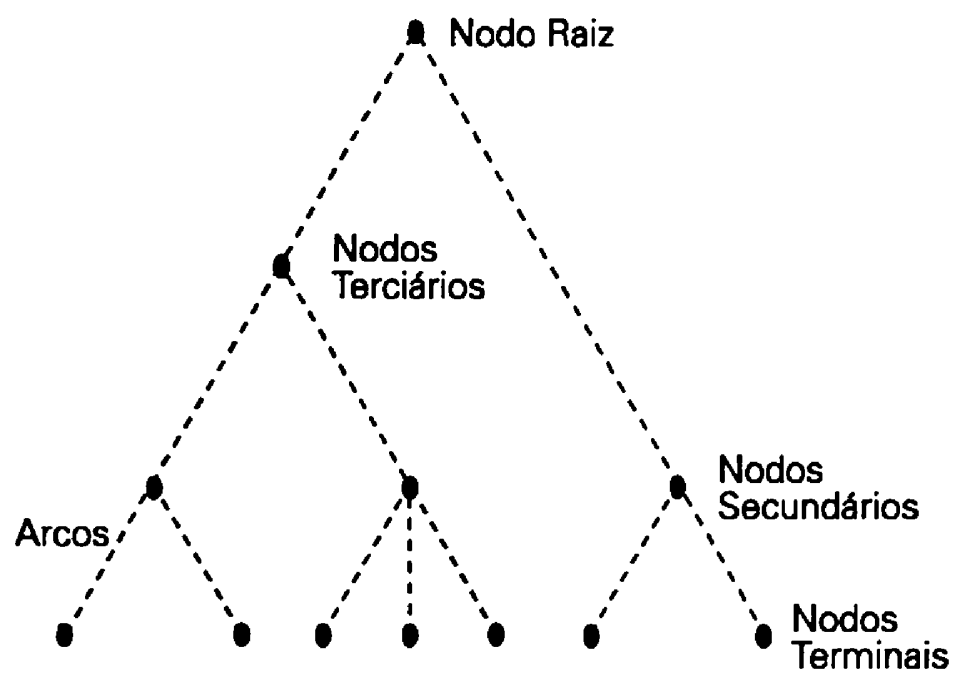


Os arcos são orientados de baixo para cima, ou seja, os nodos terminais são unidos aos nodos secundários, estes aos terciários, e assim por diante, a té o nodo raiz.

Os nodos terminais foram compostos pelos dados listados em ordem de importância. Dispostos os nodos terminais, foi pedido a ela para dar uma nota de 0 a 10, segundo sua crença quanto à importância do dado para aquele diagnóstico. Subsequentemente os nodos intermediários, ou seja, secundários, terciários, etc. foram sendo construídos de acordo com a associaçāo de dados necessários para concluir aquela hipótese diagnóstica.

A cada arco constuído foi também solicitado uma nota de 0 a 10 , até atingir o nodo raiz, quando então era lançada a nota a este nível.

O grafo foi construído pela própria enfermeira que enunciava o diagnóstico. A cada diagnóstico enunciado foi construído o grafo a ela correspondente.

A coleta de dados foi feita em duas etapas. As entrevistas foram sempre individuais e realizadas num ambiente isolado, dentro da própria UTI.

\section{2 etapa}

Na primeira etapa, todas as enfermeiras receberam a seguinte explanação introdutória:

"Quais são os diagnósticos de enfermagem específicos que você usa para os pacientes que apresentam desequilíbrio no conteúdo intracraniano? Exclua os diagnósticos referentes, por exemplo, às condiçōes que aparecem concomitantemente nesse tipo de paciente, tais como, os relacionados com a integridade cutâneo mucosa, complicaçōes pulmonares, etc. Ao formular o diagnóstico utilize aquele do seu cotidiano. Não importa se você não o considera totalmente padronizado."

Deu-se a liberdade para que cada enfermeira formulasse quantos diagnósticos quizesse, sendo que cada um deles pedia-se para construir o grafo correspondente.

Terminadas as entrevistas da $1^{\mathbf{a}}$ etapa, fez-se um intervalo de tempo para analisar a consistência dos diagnósticos elaborados pelo grupo. Definiu-se como consistente a frequência de diagnósticos iguais ou semelhantes.

\section{$2^{2}$ etapa}

Obtidos os diagnósticos mais frequentes, as mesmas enfermeiras foram novamente entrevistadas para confirmação do diagnóstico e do grafo construído.

Partindo da premissa que nos diagnósticos de enfermagem, o risco do paciente é medido segundo o potencial para o mesmo ou um risco já estabelecido, procurou-se o confronto dessas duas modalidades. Assim, se a 
enfermeira havia construído um grafo cujo diagnóstico era um risco potencial, nesta $2^{a}$ etapa ela já foi solicitada para construir o grafo do mesmo diagnóstico, porém numa situação de risco e vice-versa.

\section{RESULTADOS E DISCUSSÃO}

O estudo foi realizado com 11 enfermeiras e algumas de suas características são apresentadas no Quadro 1.

Quadro 1. Características das enfermelras segundo Id ade, tempo de experiência na UTI e cursos de especlallzação reallzados. São Paulo, 1992.

\begin{tabular}{c|c|c|l} 
Enfermaira & Idade & Exp. em UT1 & \multicolumn{1}{|c}{ Cursos de Especial izaçäo } \\
\hline 01 & 39 & 12 anos & Enf. em Terapia Intensiva \\
\hline 02 & 27 & 1 a $5 \mathrm{~m}$ & Enf. em Terapia Intensiva \\
\hline 03 & 29 & 2 a $4 \mathrm{~m}$ & Enf. em Terapia Intensiva \\
\hline 04 & 26 & 3 a $6 \mathrm{~m}$ &.. \\
\hline 05 & 34 & 8 anos & Enf. em Terapia Intensi va \\
\hline 06 & 28 & 3 anos & Enf. do Trabalho \\
\hline 07 & 40 & 12 anos & $\begin{array}{l}\text { Enf. em Terapia Intensiva } \\
\text { Administraçäo Hospitalar }\end{array}$ \\
\hline 08 & 28 & 4 anos & $\begin{array}{l}\text { Enf. em Terapia Intensiva } \\
\text { Administraçäo Hospitalar }\end{array}$ \\
\hline 10 & 27 & 2 anos & $\begin{array}{l}\text { Enf. em Terapia Intensiva } \\
\text { Enf. em Cardiologia }\end{array}$ \\
\hline 11 & 40 & 5 anos & $\begin{array}{l}\text { Enf. em Terapia Intensiva } \\
\text { Enf. Médico-cirúrgica }\end{array}$ \\
\hline
\end{tabular}

A idade média das enfermeiras foi de 32 anos e o tempo de experiência na UTI em estudo, de 5 anos. Das 11 enfermeiras, somente 2 não tinham cursos de especialização em Enfermagem em Terapia Intensiva.

$\mathrm{Na} 1^{\text {a }}$ etapa as enfermeiras construíram um total de 24 grafos cuja distribuição apresentada a seguir nos conjuntos "Potencial para..." e "Alteraçāo do(a)...". 
Tabela 1. Distribuição dos diagnósticos de enfermagem nos conjuntos "Potencial para..." e "Alteração do(a)...". São Paulo, 1992

\begin{tabular}{l|c}
\multicolumn{1}{c|}{ Diagnóstico } & $\mathbf{N}^{\mathbf{1}}$ \\
\hline Potencial para... & 15 \\
\hline Alteraçōes do (da) & $\mathbf{9}$ \\
\hline Total & 24
\end{tabular}

Cumpre mencionar que não houve limitação quanto ao número de diagnósticos por enfermeira. A cada diagnóstico que era enunciado por escrito, solicitava-se a construção do gra fo correspondente. Assim, a maioria construiu 2 a 3 grafos e duas delas, apenas 1.

Como mostra a Tabela 1 houve predomínio no conjunto "Potencial para...".

Em relação ao diagnóstico propriamente dito, a predominância recaiu nos grafos relativos à alteração do nível de consciência. Todas as enfermeiras fizeram pelo menos 1 grafo a cla relacionada, seja no conjunto "Potencial para...", seja no "Alteração do...".

Este foi o primeiro fato que chamou a atenção dado o conteúdo da questão norteadora apresentada para as enfermeiras formularem os diagnósticos de enfermagem.

MITCHELL ${ }^{8}$ propôs para tal situação o diagnóstico - diminuição da capacidade adaptativa intracraniana, tendo como etiologia a falência dos mecanismos compensatórios intracranianos normais c como caractcrísticas definidoras, os repetidos aumentos desproporcionais na pressão intracraniana (PIC) em resposta a estímulos nocivos ou não nocivos. Na ausência de monitoração da PIC o diagnóstico seria - potencial para diminuição da capacidade adaptativa intracraniana e os indicadores deste diagnóstico potencial incluiriam os resultados da tomografia computadorizada e o diagnóstico médico relacionado com a possibilidade de hipertensão intracraniana (HIC).

É preciso mencionar que esta não é uma nomenclatura aceita unanimente pelas especialistas em enfermagem em neurociência.

MILLER; WILLIAMS ${ }^{7}$ listaram diferentes rótulos diagnósticos que são utilizados para pacientes com risco ou que apresentam PIC aumentada. Dentre eles citam: "nível de resposta alterado para diminuiçāo", "alteraçāo na perfusão cerebral", "potencial para aumento de pressão intracraniana", "potencial para falência cerebral", "potencial para injúria cerebral secundária", "diminuição da capacidade adaptativa intracraniana". Alertam para a falta de características definidoras específicas, consistentes ou padronizadas. Após analisarem o diagnóstico "alteração na perfusão cerebral"propuseram um outro alternativo que seria "PIC aumentada relacionada a estímulos específi- 


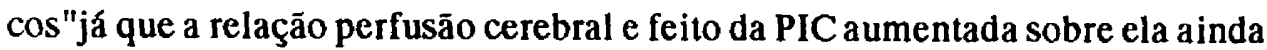
nāo é muito clara.

No presente estudo, os diagnósticos foram formulados como "Potencial para alteração do nível de consciência relacionados com..." ou "alteração no nível de consciência relacionado com..."sendo que nesta relação causual é que se definiu o paciente com PIC aumentada, como será discutido mais adiante.

Identificado o diagnóstico predominante, como mencionado na metodologia, a $2^{\mathrm{a}}$ etapa de coleta de dados foi retomada a partir de grafo construído na $1^{a}$ etapa. Assim, se aquela enfermeira havia construído um grafo relativo a "Potencial para alteraçāo do nível de consciência relacionado com..." era pedido o grafo "alteração do nível de consciência relacionado com..." e vice-versa. Tomou-se sempre o cuidado de confirmar com cada uma delas se o grafo elaborado anteriormente corresponde ao diagnóstico enunciado no que se referia ao risco.

Desta forma, foram obtidos 11 grafos para o conjunto "potencial para..." e 11 para o "Alteração do..." que se distribuiram como se segue.

Tabela 2. Distribuição dos diagnósticos "Potencial para..." e "Alteração no nivel de consciência" segundo sua relação causual. São Paulo, 1992.

\begin{tabular}{l|c}
\multicolumn{1}{c|}{ Relaçāo Causal } & $\mathrm{N}^{\circ}$ \\
\hline Compressāo de estrutura encefálica & 8 \\
\hline Distúrbio na perfusāo cerebral & 2 \\
\hline Indefinido & 1 \\
\hline Total & 11
\end{tabular}

Nesta fase, evidenciou-se com maior clareza que a forma de redigir o diagnóstico era uniforme e que a maioria apontou como relação causual a compressão de estruturas encefálicas. Todavia, esta relação causal estava descrita de formas variadas, tais como, herniação, prejuízo no tecido cerebral, aumento da PIC, presença de hematoma, tumor ou trauma, sofrimento cerebral, compressão cerebral, edema cerebral.

Embora esta frequência seja baixa, os grafos construídos por 2 enfermeiras e que relacionaram-se com distúrbios na perfusāo cerebral, também tendiam para a diversificação.

A especificidade da relação causal parece ser o fator que ocasionou a grande diversificação dos nodos terminais nos grafos. Dada a diversificação, não foi possível construir grafos médios desses diagnósticos.

Entretanto a título de ilustração, apresenta-se alguns grafos construídos para o diagnóstico "Potencial para alteração do nível de consciência...". 

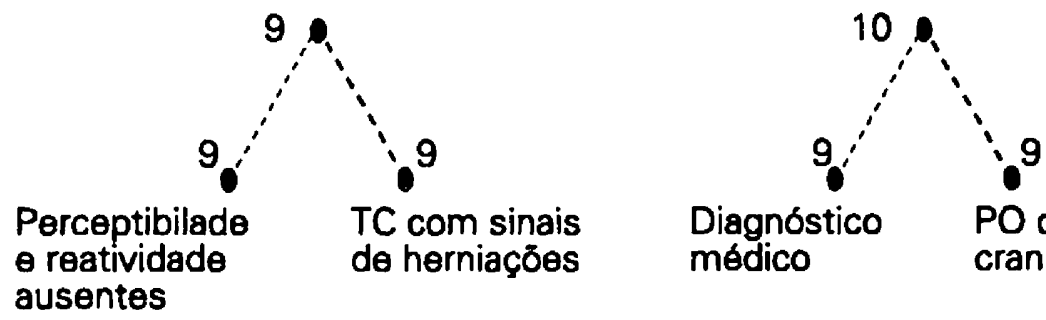

\section{Perceptibilade ausentes}

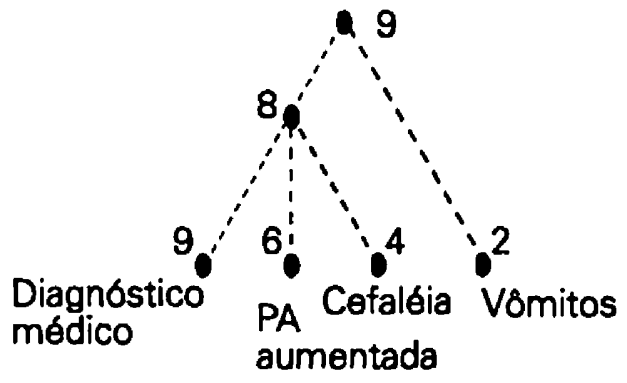

Como pode-se verificar, em geral, trata-se de grafos bastante simples com uma hierarquia de nodo intermediário ou passagem direta para o nodo raiz. Contudo, a dispersão dos nodos terminais foi muito grande.

Já, nos diagnósticos "Alteração no nível de consciência...", geralmente, o grafo foi constituído com duas hierarquias de nodos intermediários. Como no conjunto anterior, a dispersão no primeiro nível era evidente tanto naqueles com maior ou menor número de nodos intermediários.

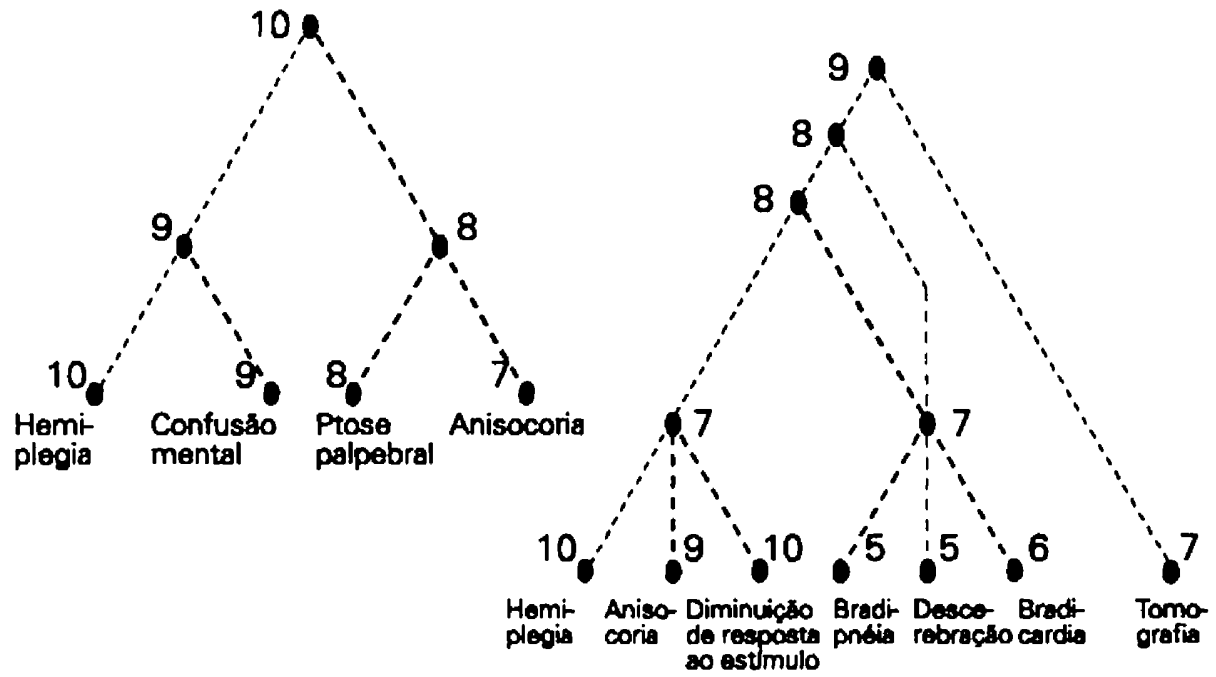


A diversidade dos dados que compuseram os nodos terminais pode ser vista nas Tabelas 3 e 4 .

Tabela 3. Dados que compuseram os nodos terminais e suas frequências no diagnóstico "Potencial para alteração no nível de consciência relacionado com compressão das estruturas encefálicas". São Paulo, 1992.

\begin{tabular}{l|c}
\multicolumn{1}{c|}{ Dados dos Nodos Termi nais } & $N^{\circ}$ \\
\hline Diagnóstico médico & 4 \\
\hline Tomografia oom si nais de hemiaçāo & 4 \\
\hline Aumento da pressāo arterial & 3 \\
\hline Hemi plegia/hemi paresia/déficit motor & 3 \\
\hline Cefaléia & 2 \\
\hline Vômitos & 2 \\
\hline Dimi nuiçāo no nivel de consciência & 2 \\
\hline Perceptividade e reatividade ausentes & 1 \\
\hline Di plopia & 1 \\
\hline Alteraçāo na respiraçāo & 1 \\
\hline Fratura de ossos cranianos & 1 \\
\hline Gasometria alterada & 1 \\
\hline Pós craniotomia & 1 \\
\hline Total & 26 \\
\hline
\end{tabular}

Considerando-se que neste conjunto foram construídos 8 grafos, a média de nodos terminais por diagnóstico "Potencial..." foi aproximadamente 3. 
Tabela 4. Dados que compuseram os nodos terminais e suas frequências no diagnóstico "Alteração no nível de consciência relacionado com compressão de estruturas encefálicas". São Paulo, 1992.

\begin{tabular}{|c|c|}
\hline Dados dos Nodos Terminais & $\mathrm{N}^{\circ}$ \\
\hline Anisocoria & 5 \\
\hline Ausência ou dimi nuiçāo de respostas a estímul os & 3 \\
\hline Hemi plegia/hemiparesia/déficit motor & 3 \\
\hline Confusāo mental & 3 \\
\hline Reflexo fotomotor e ocul ocefálico a usente & 2 \\
\hline Cefaléia & 2 \\
\hline Aumento da pressāo arterial & 2 \\
\hline Nauseas/vômi tos & 2 \\
\hline Resul tados da tomografia cerebral & 2 \\
\hline Apnéi a/bradipnéia & 2 \\
\hline Descerebraçāo/decorticaçāo & 2 \\
\hline Diagnóstico médico & 1 \\
\hline Diplopia & 1 \\
\hline Ptose palpebral & 1 \\
\hline Aumento da PIC & 1 \\
\hline Desvio conjugado do olhar & 1 \\
\hline Rigidez de nuca & 1 \\
\hline Babinsky positi vo & 1 \\
\hline Abrir $\propto$ ol olhos aos estímul os dolorosos & 1 \\
\hline Tontura & 1 \\
\hline Total & 37 \\
\hline
\end{tabular}

Igualmente, neste conjunto, foram construídos 8 grafos. Portanto, a média de nodos terminais por diagnóstico "Alteração..." foi aproximadamente 5.

GRANT; KINNEY ${ }^{2}$ analisaram a validade do diagnóstico de enfermagem - alteração no nível de consciência, tendo como estrutura conceitual o raciocínio diagnóstico. Na $1^{\text {a }}$ fase, utilizando a técnica de Delphi, 26 enfermeiros em neurociência, definiram as características e a operacionalização deste diagnóstico e a dividiram em: alteração do nível de consciência relacionada 
com despertar e alteração do nível de consciência relacionado com conteúdo mental. Na $2^{\mathrm{a}}$ fase, 30 enfermeiros de UTI de neurologia, estimaram a importância e a frequência desses dois diagnósticos e identificaramas características definidoras mais importantes e menos importantes. $\mathrm{Na} 3^{\mathrm{a}}$ fase, esses diagnósticos foram validados em 60 pacientes avaliados quanto à ocorrência dessas características de acordo com a pontuação da escala de coma de Glasgow.

Um fato que chama a atenção, no presente estudo, é que nenhuma das enfermeiras entrevistadas abordou o diagnóstico "alteraçāo no nível de consciência" desta forma, ou seja, ligado diretamente ao que se altera - despertar ou conteúdo mental. Outrossim, o controle da alteração no nível de consciência nesta UTI, é feito pela escala de Glasgow.

A direção tomada por 8 das 11 enfermeiras foi para desequilíbrio no conteúdo intracraniano.

GRANT; KINNEY ${ }^{3}$ ao ilustrar as características definidoras e a operacionalizaçāo do diagnóstico "alto risco de injúria cerebral secundária" apresenta dados que, parecem estar mais próximos daqueles obtidos neste estudo. As autoras, apresentaram uma lista parcial das características definidoras: confusāo, desorientação, reação pupilar alterada, anisocoria, atividade reflexa tronco-cerebral diminuida, decorticação, padrāo respiratório alterado, prostração. Completam o diagnóstico como "alto risco para injúria cerebral secundária devido à hipoxia, hipercapnia, hipertermia ou estímulo ambiental excessivo"e que o fator etiologico poderia ser, por exemplo. o paciente com trauma craniano fechado.

Como pode-se depreender há ainda muito a definir em relação ao rótulo diagnóstico de enfermagem, assim como, as bases que a fundamentam, sem falar nas intervenções que dela advém e que compõem o raciocínio diagnóstico global. Contudo, uma estrutura de conhecimentos em desenvolvimento pode ser constatada tanto neste estudo, como na literatura existente sobre o assunto.

Ressalte-se que a população deste estudo foi constituída, principalmente, por enfermeiras com especialização em terapia intensiva (Quadro 1), e não enfermagem em neurociência. Todas possuiam uma vivência no cuidado com pacientes que apresentam desequilíbrio no conteúdo intracraniano mas, o referencial para o diagnóstico de enfermagem, conforme pôde ser constatado durante as entrevistas, não era especificamente o que vem sendo investigado pelas enfermeiras de neurociência.

A uniformidade em enunciar o diagnóstico de enfermagem, mostra coesão e provavelmente, reciclagem para padronização. Comentários como nunca ter refletido como chegava àquele diagnóstico foi frequente, assim como, a observaçāo de um certo grau de surpresa pela facilidade em construir o grafo. Isto mostra que na prática, o raciocínio diagnóstico vem sendo desenvolvido. 
Desta forma, exercitar como estão construindo seus diagnósticos, talvez com o uso deste método, seja um dos caminhos para elucidaçāo das caractcrísticas definidoras e operacionalização dos diagnósticos de enfermagem.

\section{CONCLUSÕES E RECOMENDAÇÖES}

Respeitadas as limitações destc estudo c o número reduzido de enfermeiras que dele participaram, constatou-se:

- estrutura de conhecimento em desenvolvimento,

- uniformidade quanto ao enunciado dos diagnósticos de enfermagem,

- diversificação quanto à base de dados que compõem os diagnósticos ligada diretamente com a forma de especificação da relação causal.

Além disso, o método de aquisiçāo de conhecimentos utilizado, mostrou ser bastante promissor também para esta análise, sobretudo pela facilidade de instrumentalização. Recomenda-se, portanto, quc estudos que ampliem e aprofundem esta análise sejam efetuados.

KOIZUMI, M.S. et al. A method to analyse clinical reasoning applied in neuroscience nursing. Rev. Esc. Enf. USP., v. 27, n. 1, p. 161-73 , apr. 1993.

It is a preliminary study testing the applicability of a method for knowledge characterization based on graphs theory. 11 (eleven) nurses working in a medical surgical intensive care unit including neurological patients contructed graphs to explain the diagnostic process. The results show that: the knowledge structure is in a developing stage; there is agreement regarding to diagnosis formulation and the data base on which it is founded is diversified and link to a specified form of related causes. The method demonstrated to be feasible and should be reapplied on a larger population.

UNITERMS: Neurological nursing. Nursing Diagnosis.

\section{REFERÊNCIAS BIBLIOGRÁFICAS}

1. AMERICAN NURSE'S ASSOCIATION/AMERICAN ASSOCIATION OF NEUROSCIENCE NURSES. Neuroscience nursing practice: process and outcome criteria of selected diagnoses. Kansas City, 1985. (Publ. MS-13).

2. GRANT, J.S.; KINNEY, M. Altered level of consciousness: validity of a nursing diagnoses Res. Nurs. Health, v. 13, n. 6, p. 403-10, 1990.

3. GRANT, J.S.; KINNEY, M. Cinical referents for nursing diagnoses. L.Neuroscience Nurs., v. 24, n. 2, p. 94-8, 1992.

4. GRECO, G.; ROCHA, A.F. The fuzzy logic of text understanding. Fuzzy Sets and Systems v. 33, p. 347-60, 1987.

5. LEÇO, B. de F. Construgão da base de conhecimente de um sistema especialista de apoio ao diagnóstico de candiopatias congênitas. São Paulo, 1988. Tese (Doutorado) - Escola Paul ista de Medicina.

6. MACHADO, R.J. et al. Calculating the mean knowledge representation from multiple experts. In: KACPRZYK, J.; FREDRIZZ, M. Multiperson decision making using fuzzi sets and possibility theory. Netherlandas, Kluwer Academi c, 1990. p. 113-27. 
7. MILJER, E; WILLJAMS, S. Alteration in cerebral perfusion: clinical concept or nursing diagnosis? J.Neurosci Nurs., v.19, n. 4, p. 183-90, 1987.

8. MITCHELh, P.H. Decreased adaptative capacity intracranial: a proposal for a nursing diagnosis. L. Neurescience Nurs., v. 18, n. 4, p. 170-5, 1986.

9.MITCHELL, P.H. et al. ASNN'S neuroscience nursing phenomena and practice. Norwalk, Appleton \& Lange, 1988.

10. ROCHA, AF. da; ROCHA, M.T. Specialized speech: a first prose for language expert systems. Inf.Sci. v. 35, p. 1-8, 1985.

11. ROCHA, M.T. Decorficacajo de um texto sobre hanseniase por estudantes, docentes e pessoal deenfermagem. Sāo Paulo, 1990. Tese (Doutorado) - Facul dade de Saúde Pública, Uni versidade de Sāo Paulo. 